\title{
Vibrational and Electronic Structure of the $\alpha$ - and $\beta$-Naphthyl Radicals via Slow Photoelectron Velocity-Map Imaging
}

\author{
Marissa L. Weichman, ${ }^{\dagger}$ Jongjin B. Kim, ${ }^{\dagger}$ Jessalyn A. DeVine, ${ }^{\dagger}$ Daniel S. Levine, ${ }^{\dagger}$ \\ and Daniel M. Neumark ${ }^{* \dagger,}+$ \\ ${ }^{\dagger}$ Department of Chemistry, University of California, Berkeley, California 94720, United States \\ ${ }^{\ddagger}$ Chemical Sciences Division, Lawrence Berkeley National Laboratory, Berkeley, California 94720, United States
}

Supporting Information

ABSTRACT: Slow photoelectron velocity-map imaging (SEVI) spectroscopy has been used to study the vibronic structure of gas-phase $\alpha$ - and $\beta$-naphthyl radicals $\left(\mathrm{C}_{10} \mathrm{H}_{7}\right)$. SEVI of cryogenically cooled anions yields spectra with $<4$ $\mathrm{cm}^{-1}$ resolution, allowing for the observation and interpretation of congested vibrational structure. Isomerspecific photoelectron spectra of detachment to the radical ground electronic states show detailed structure, allowing assignment of vibrational fundamental frequencies. Transitions to the first excited states of both radical isomers are also observed; vibronic coupling and photodetachment threshold effects are considered to explain the structure of the excited bands.

$\mathrm{P}$ olycyclic aromatic hydrocarbons (PAHs) are of major importance in wide-ranging areas of chemistry. PAHs are involved in the combustion of organic matter ${ }^{1}$ and subsequent soot formation, ${ }^{2,3}$ while neutral, ionized, hydrogenated, and dehydrogenated PAHs are likely constituents of the interstellar medium (ISM). ${ }^{4,5}$ PAHs are possible sources of mid-infrared emission features in the ISM $^{6-8}$ and have been considered tentative candidates for carriers of diffuse interstellar bands (DIBs) for many years. ${ }^{9-11}$ No conclusive evidence of small PAHs as DIB carriers exists to date, despite much work searching for matches between laboratory spectra and astronomical data. ${ }^{12,13}$ Decomposition of large interstellar PAHs may lead to the formation of carbon chains and hydrocarbon radicals in space. ${ }^{14}$

In this Communication, we report high-resolution anion photoelectron spectra of $\alpha$ - and $\beta$-naphthyl, $\mathrm{C}_{10} \mathrm{H}_{7}^{-}$, whose structures are shown in Figure 1. Naphthalene is the simplest $\mathrm{PAH}$; its derivatives are therefore tractable models for the behavior of larger aromatic systems.

There is a solid body of theoretical work on the electronic structure, geometries, and vibrations of the naphthyl radicals and anions ${ }^{5,15-20}$ and some calculations of their reactivity in the context of combustion. ${ }^{21,22}$ Experimental characterization is sparser. Reed and Kass ${ }^{23}$ and Lardin et al. ${ }^{24}$ measured the electron affinities (EAs) of the $\alpha$ - and $\beta$-naphthyl radicals through kinetic methods and calculated the $\alpha$ and $\beta \mathrm{C}-\mathrm{H}$ bond dissociation energies of naphthalene. Both studies found the $\alpha$ anion to be lower in energy than the $\beta$ anion by several $\mathrm{kJ} / \mathrm{mol}$, favoring $\alpha$ formation in deprotonation of naphthalene.
Anion photoelectron spectroscopy (PES) is a powerful technique for probing the vibronic structure of neutral radicals through photodetachment of a closed-shell anion. ${ }^{25,26}$ Ervin et al. ${ }^{18}$ measured the photoelectron spectrum of $\mathrm{C}_{10} \mathrm{H}_{7}{ }^{-}$at $300 \mathrm{~K}$ and with a resolution of $\sim 100 \mathrm{~cm}^{-1}$. The authors reported a congested, partially resolved spectrum of the radical ground state with an EA of 1.403(15) eV. By comparison to FranckCondon (FC) simulations, the spectrum was assigned to an 11:1 $\alpha: \beta$ isomer ratio. Substantial enrichment in $\alpha$-naphthyl is consistent with the authors' use of nonspecific deprotonation of naphthalene to generate anions.

Slow photoelectron velocity-map imaging (SEVI) spectroscopy, ${ }^{25}$ a high-resolution variant of anion PES, is a method apt to tackle the challenges of the naphthyl system in combination with gas-phase synthesis techniques and ion cooling. When anions are cryogenically cooled prior to photodetachment, hot bands and sequence bands vanish and rotational envelopes dramatically narrow, yielding features as narrow as $4 \mathrm{~cm}^{-1}$ full width at half-maximum (fwhm) for molecular systems. ${ }^{27}$ We report vibrationally resolved, isomer-specific SEVI spectra of the ground and first excited states of the $\alpha$ - and $\beta$-naphthyl radicals, providing a wealth of vibronic information on these species.

The SEVI method and apparatus are described in detail elsewhere. $^{25,27,28}$ Naphthyl anions were prepared by flowing trace $\mathrm{NF}_{3}$ in helium gas over a reservoir containing $\alpha$ - or $\beta$ trimethylsilyl (TMS)-naphthalene, and expanding this mixture through an Even-Lavie pulsed valve ${ }^{29}$ fitted with a circular filament ionizer. The TMS-naphthalene precursors were synthesized according to the procedure described by Marcinow et al. $^{30}$ from the corresponding $\alpha$ - and $\beta$-bromonaphthalenes (Sigma-Aldrich, 97\%). Dissociative electron attachment to $\mathrm{NF}_{3}$ produced $\mathrm{F}^{-}$which reacted with TMS-naphthalene, selectively forming the $\alpha$ - or $\beta$-naphthyl anion. ${ }^{23,31}$ The anions were collisionally cooled in an ion trap held at $5 \mathrm{~K}$, mass-selected, and then photodetached with the output from a tunable dye laser. The electron kinetic energy ( $\mathrm{eKE}$ ) distribution of the resulting photoelectrons was measured with a velocity-map imaging spectrometer operated using low extraction voltages to preferentially detect low-eKE electrons, which are imaged with the highest resolution.

Received: December 8, 2014

Published: January 20, 2015 


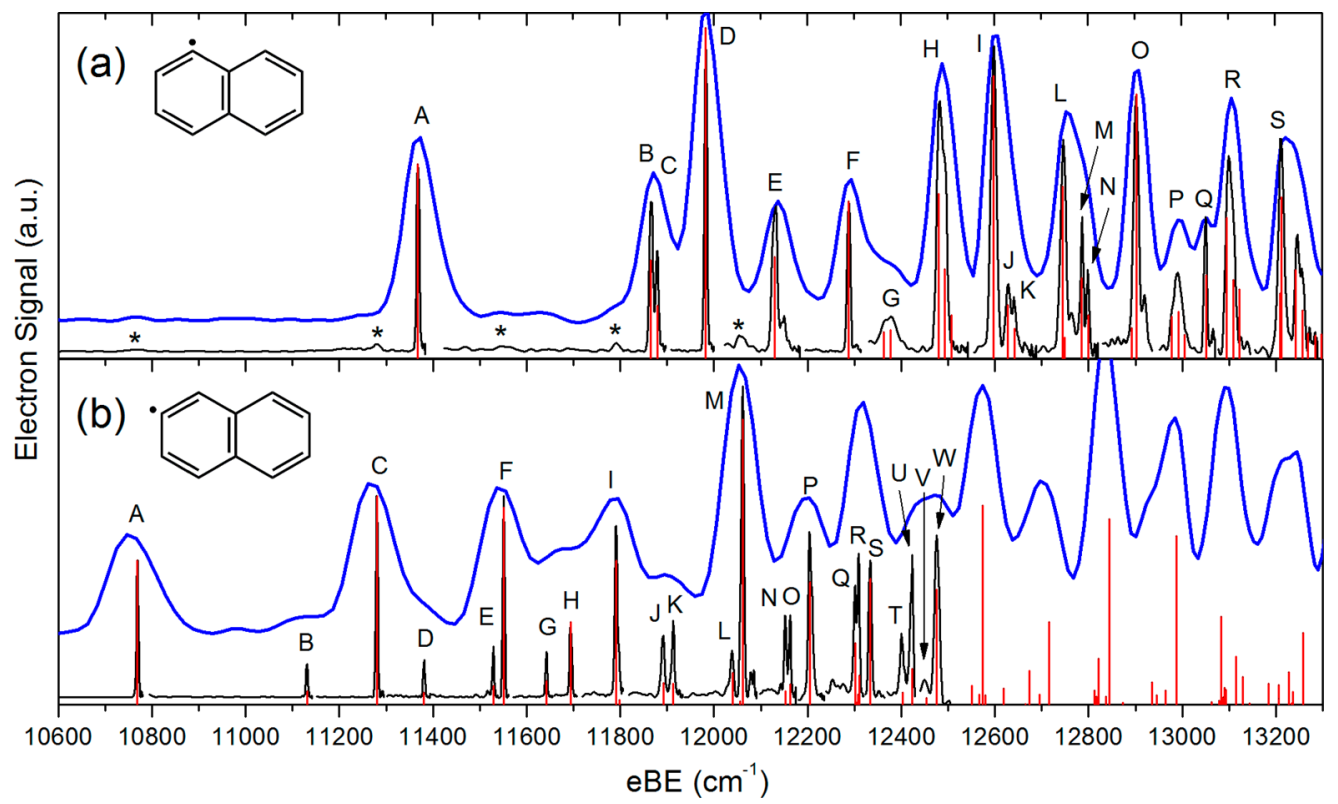

Figure 1. SEVI spectra of photodetachment to the $\tilde{X}^{2} A^{\prime}$ ground states of (a) $\alpha$-naphthyl and (b) $\beta$-naphthyl, with overview scans in blue, highresolution traces in black, and FC simulations in red.

Energetics, optimized $C_{s}$ geometries and harmonic frequencies were calculated for the $\alpha$ - and $\beta$-naphthyl anionic and neutral states at the B3LYP/6-311+G* level of theory, which has previously worked well for PAH systems. ${ }^{32}$ Calculations of excited state geometries and harmonic frequencies were carried out with the maximum overlap method (MOM). ${ }^{33}$ Dyson orbitals were calculated with EOM-IP-CCSD/6-311+G*. All calculations were done in Q-Chem 4.0. ${ }^{34,35}$ Zero-point corrected energetics and selected vibrational frequencies are reported in Table 1. Complete lists of calculated geometries and frequencies are reported in Tables $\mathrm{S} 1-\mathrm{S} 4$ of the Supporting Information (SI), with molecular structures labeled in Figure S1.

Table 1. Experimental and Calculated Electron Affinities, Term Energies, and Vibrational Frequencies for the $\alpha$ - and $\beta$-Naphthyl Radicals

\begin{tabular}{|c|c|c|c|c|}
\hline & & & exptl & calcd \\
\hline \multirow[t]{7}{*}{$\alpha$} & $\tilde{X}$ & $\mathrm{EA},{ }^{a} \mathrm{eV}$ & $1.4095(4)^{b}$ & 1.3811 \\
\hline & & $\nu_{25}, \mathrm{~cm}^{-1}$ & $921(3)$ & 933 \\
\hline & & $\nu_{27}, \mathrm{~cm}^{-1}$ & $763(5)$ & 773 \\
\hline & & $\nu_{28}, \mathrm{~cm}^{-1}$ & $615(3)$ & 632 \\
\hline & & $\nu_{29}, \mathrm{~cm}^{-1}$ & $512(3)$ & 521 \\
\hline & & $\nu_{30}, \mathrm{~cm}^{-1}$ & $498(4)$ & 508 \\
\hline & $\tilde{A}$ & $\mathrm{TE},{ }^{c} \mathrm{eV}$ & $1.7778(32)$ & 1.6269 \\
\hline \multirow[t]{8}{*}{$\beta$} & $\tilde{X}$ & EA, eV & $1.3352(2)$ & 1.3266 \\
\hline & & $\nu_{25}, \mathrm{~cm}^{-1}$ & $925(3)$ & 937 \\
\hline & & $\nu_{26}, \mathrm{~cm}^{-1}$ & $782(2)$ & 794 \\
\hline & & $\nu_{27}, \mathrm{~cm}^{-1}$ & $760(2)$ & 767 \\
\hline & & $\nu_{28}, \mathrm{~cm}^{-1}$ & $612(2)$ & 629 \\
\hline & & $\nu_{29}, \mathrm{~cm}^{-1}$ & $511(2)$ & 520 \\
\hline & & $\nu_{31}, \mathrm{~cm}^{-1}$ & $362(2)$ & 369 \\
\hline & $\tilde{A}$ & $\mathrm{TE}, \mathrm{eV}$ & $2.0194(27)$ & 1.8652 \\
\hline
\end{tabular}

${ }^{a}$ Electron affinity. ${ }^{b}$ Uncertainties represent one standard deviation of a Gaussian fit to the experimentally observed peak. ${ }^{c}$ Term energy.
FC simulations were carried out using ezSpectrum, ${ }^{36}$ with all modes treated in the harmonic approximation and with full Duschinsky mixing. Simulations were shifted in binding energy and scaled to align with the position and intensity of each experimental vibrational origin. Vibrational fundamentals that were observed in the SEVI spectra were scaled to their experimental values; the others were left at their B3LYP calculated values. Photodetachment cross sections were calculated as a function of eKE using the ezDyson program. ${ }^{37,38}$ Given $a b$ initio Dyson orbitals, ezDyson finds the contribution of partial spherical waves with angular momentum $l \leq 4$ to the wave function of the outgoing photoelectron. Photoelectron angular distributions were also considered, as discussed in the SI.

SEVI spectra of photodetachment to the low-eBE edge of the $\alpha$ - and $\beta$-naphthyl radical $\tilde{X}^{2} A^{\prime}$ bands are shown in Figure 1 . Low-resolution spectra are plotted in blue while high-resolution composite traces are in black. Overview spectra of the entire FC profile of the $\tilde{X}$ bands for the two isomers are given in Figure S2. High-resolution peak widths are $\sim 8 \mathrm{~cm}^{-1} \mathrm{fwhm}$, in line with typical SEVI resolution for molecular systems. The ground state spectra of both isomers show congested FC activity that is accurately predicted by FC simulations (red). Peaks indicating a $\sim 4 \%$ contamination of the $\beta$ isomer in the $\alpha$-naphthyl spectrum are marked with asterisks in Figure 1a, and are consistent with the purity of the $\alpha$-bromonaphthalene starting material and ${ }^{1} \mathrm{H}$ NMR characterization of the $\alpha$-TMS-naphthyl precursor.

Spectra of the $\tilde{A}^{2} A^{\prime \prime}$ excited states of the two isomers are shown in Figure 2. The $\tilde{A}$ bands of both isomers have strong vibrational origins and weak vibrationally excited FC structure, qualitatively matched by simulation. The $\tilde{A}$ state vibrational origins have vanishing intensities close to threshold. SEVI can only yield narrow features at low eKE, and thus origin peak widths are limited to $\sim 60 \mathrm{~cm}^{-1}$ fwhm by the experimental resolution. 


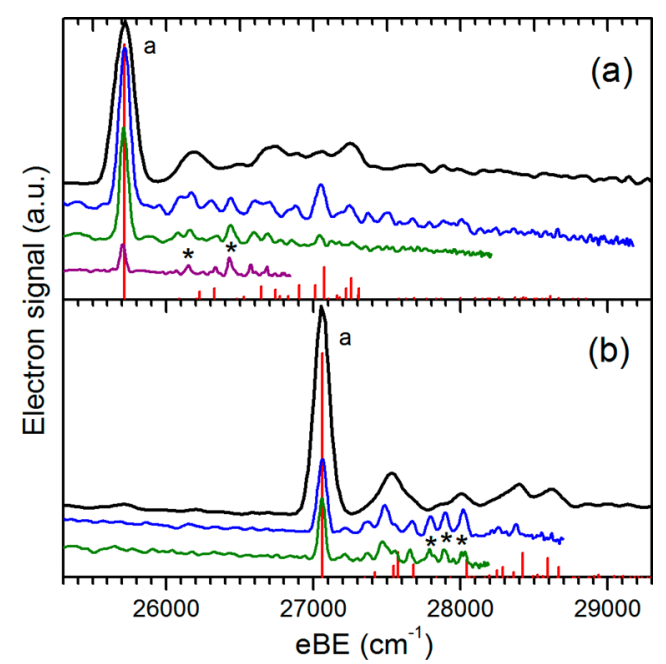

Figure 2. SEVI spectra and FC simulation of photodetachment to the $\tilde{A}^{2} A^{\prime \prime}$ first excited states of (a) $\alpha$-naphthyl and (b) $\beta$-naphthyl, showing overview spectra in black, FC simulations in red, and colored traces taken at progressively lower photon energies.

Experimental findings are summarized in Table 1, and compare well to calculated values. Full spectral assignments can be found in Tables S5 and S6.

The spectra shown in Figure 1 represent the first highresolution characterization of the $\alpha$ - and $\beta$-naphthyl radical ground states, and are a testament to the efficacy of the SEVI method when combined with cryogenic cooling and isomer selection techniques. Our overview spectrum of the $\alpha$ - naphthyl $\tilde{X}$ band (Figure S2) is in excellent agreement with the naphthyl spectrum reported by Ervin et al. ${ }^{18}$ Our measured EAs for $\alpha$ and $\beta$-naphthyl (Table 1) agree with prior work, with much improved precision. ${ }^{18,23,24}$

Both $\tilde{X}$ bands show extensive FC activity, indicative of a large change in geometry upon photodetachment. For these transitions, the electron is detached from an s-p hybrid orbital localized on the deprotonated site; calculated Dyson orbitals are shown in Figure 3. Correspondingly, geometry optimization

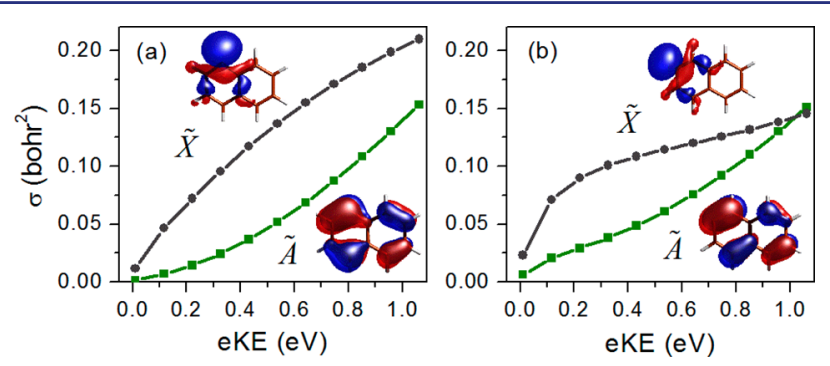

Figure 3. Calculated cross sections and visualized Dyson orbitals for photodetachment to the $\tilde{X}$ and $\tilde{A}$ states of (a) $\alpha$-naphthyl and (b) $\beta$ naphthyl as a function of eKE.

calculations indicate that for both anions, the $\mathrm{C}-\mathrm{C}-\mathrm{C}$ interior bond angle at the deprotonated site changes dramatically upon photodetachment, narrowing by $14^{\circ}$ (Tables S1 and S2). The highly FC active vibrational modes are in-plane, as required by the $C_{s}$ symmetry of the system, and involve distortion at the deprotonated site (Figure S3).

The SEVI spectra of the $\alpha$-and $\beta$-naphthyl $\tilde{A}$ bands shown in Figure 2 represent the first experimental report of these states. The observed term energies are well matched by MOM calculations (Table 1). The $\tilde{A}$ bands of both species have intense vibrational origins, indicating small changes in geometry upon photodetachment. Accordingly, the calculated excited state geometries show little displacement from the anion geometries (Tables S1 and S2), and the Dyson orbitals for these transitions are highly delocalized (Figure 3 ).

The poor resolution of the $\tilde{A}$ bands results from transition cross sections decaying close to threshold. According to the Wigner threshold law, ${ }^{39} \sigma \propto(\mathrm{eKE})^{l+1 / 2}$, where $\sigma$ is the nearthreshold photodetachment cross section and $l$ is the angular momentum of the nascent photoelectron. Considering the naphthyl isomers as pseudo- $D_{2 h}$ species, the $\tilde{A}$ state Dyson orbitals have $A_{u}$ symmetry. Within the s\&p model, ${ }^{40}$ an $A_{u}$ photodetachment transition produces outgoing electrons with $l$ $\geq 2$, yielding vanishing $\sigma$ at small eKE. The $\tilde{X}$ states, on the other hand, have localized s-p hybrid Dyson orbitals, which allows for partial $l=0$ detachment and retained intensity at small eKE. ${ }^{41}$ Calculated photodetachment cross sections, shown in Figure 3, are consistent with this threshold behavior for the $\tilde{X}$ and $\tilde{A}$ states of both species.

Some weak vibrational features (marked with asterisks in Figure 2) maintain intensity close to threshold in the $\tilde{A}$ state spectra of both species, and are not well-represented by simulation. We assign these features as transitions to FCforbidden $a^{\prime \prime}$ vibrational modes of the $\tilde{A}^{2} A^{\prime \prime}$ state, which can undergo Herzberg-Teller coupling to $a^{\prime}$ vibrational levels of the $\tilde{X}^{2} A^{\prime}$ ground state. This coupling allows otherwise forbidden $\tilde{A}$ state $a^{\prime \prime}$ modes to borrow threshold behavior from the $\tilde{X}$ state, explaining both why these features do not appear in simulations, and why they retain intensity at low eKE. More detailed discussion of vibronic coupling among neutral electronic states in SEVI experiments can be found in ref 32 .

The present work represents the first high-resolution spectroscopic characterization of the ground states of the $\alpha$ and $\beta$-naphthyl radicals, and the first experimental measurement of the lowest excited states of both species. This study demonstrates the utility of SEVI for studying such species, especially when combined with cryogenic cooling and synthetic techniques for isomer selection.

\section{ASSOCIATED CONTENT}

\section{Supporting Information}

Full list of experimental spectral peak positions and assignments, calculated geometries, and vibrational frequencies for all relevant species, molecular structures, overview spectra, schematics of vibrational normal mode displacements, and discussion of photoelectron angular distributions. This material is available free of charge via the Internet at http://pubs.acs.org.

\section{AUTHOR INFORMATION}

\section{Corresponding Author}

*dneumark@berkeley.edu

\section{Notes}

The authors declare no competing financial interest.

\section{ACKNOWLEDGMENTS}

This work was supported by the Director, Office of Basic Energy Sciences, Chemical Sciences Division of the U.S. Department of Energy under Contract No. DE-AC0205CH11231. M.L.W. and D.S.L. thank the National Science Foundation for graduate research fellowships. 


\section{REFERENCES}

(1) Fialkov, A. B.; Dennebaum, J.; Homann, K.-H. Combust. Flame 2001, 125, 763.

(2) Richter, H.; Howard, J. B. Prog. Energy Combust. Sci. 2000, 26, 565.

(3) Frenklach, M. Phys. Chem. Chem. Phys. 2002, 4, 2028.

(4) Omont, A. Astron. Astrophys. 1986, 164, 159.

(5) Falvo, C.; Friha, H.; Pino, T.; Dhaouadi, Z.; Parneix, P.; Calvo, F.; Bréchignac, P. Phys. Chem. Chem. Phys. 2013, 15, 10241.

(6) Puget, J. L.; Léger, A. Annu. Rev. Astron. Astrophys. 1989, 27, 161.

(7) Allamandola, L. J.; Tielens, A. G. G. M.; Barker, J. R. Astrophys. J. Suppl. S 1989, 71, 733.

(8) Tielens, A. G. G. M. Annu. Rev. Astron. Astrophys. 2008, 46, 289.

(9) Léger, A.; d'Hendecourt, L. Astron. Astrophys. 1985, 146, 81.

(10) Ruiterkamp, R.; Halasinski, T.; Salama, F.; Foing, B. H.; Allamandola, L. J.; Schmidt, W.; Ehrenfreund, P. Astron. Astrophys. 2002, 390, 1153.

(11) Salama, F.; Galazutdinov, G. A.; Krełowski, J.; Biennier, L.; Beletsky, Y.; Song, I.-O. Astrophys. J. 2011, 728, 154.

(12) Galazutdinov, G.; Lee, B.-C.; Song, I.-O.; Kazmierczak, M.; Krełowski, J. Mon. Not. R. Astron. Soc. 2011, 412, 1259.

(13) Searles, J. M.; Destree, J. D.; Snow, T. P.; Salama, F.; York, D. G.; Dahlstrom, J. Astrophys. J. 2011, 732, 50.

(14) Pety, J.; Teyssier, D.; Fossé, D.; Gerin, M.; Roueff, E.; Abergel, A.; Habart, E.; Cernicharo, J. Astron. Astrophys. 2005, 435, 885.

(15) Pauzat, F.; Talbi, D.; Ellinger, Y. Astron. Astrophys. 1995, 293, 263.

(16) Du, P.; Salama, F.; Loew, G. H. Chem. Phys. 1993, 173, 421.

(17) Curran, H.; Wu, C.; Marinov, N.; Pitz, W. J.; Westbrook, C. K.; Burcat, A. J. Phys. Chem. Ref. Data 2000, 29, 463.

(18) Ervin, K. M.; Ramond, T. M.; Davico, G. E.; Schwartz, R. L.; Casey, S. M.; Lineberger, W. C. J. Phys. Chem. A 2001, 105, 10822.

(19) Papas, B. N.; Wang, S.; DeYonker, N. J.; Woodcock, H. L.; Schaefer, H. F. J. Phys. Chem. A 2003, 107, 6311.

(20) Kokkin, D. L.; Schmidt, T. W. J. Phys. Chem. A 2006, 110, 6173.

(21) Wang, H.; Frenklach, M. Combust. Flame 1997, 110, 173.

(22) Zhou, C.-W.; Kislov, V. V.; Mebel, A. M. J. Phys. Chem. A 2012, 116,1571

(23) Reed, D. R.; Kass, S. R. J. Mass Spectrom. 2000, 35, 534.

(24) Lardin, H. A.; Squires, R. R.; Wenthold, P. G. J. Mass Spectrom. 2001, 36, 607 .

(25) Neumark, D. M. J. Phys. Chem. A 2008, 112, 13287.

(26) Lineberger, W. C. Annu. Rev. Phys. Chem. 2013, 64, 21.

(27) Hock, C.; Kim, J. B.; Weichman, M. L.; Yacovitch, T. I.; Neumark, D. M. J. Chem. Phys. 2012, 137, 244201.

(28) Osterwalder, A.; Nee, M. J.; Zhou, J.; Neumark, D. M. J. Chem. Phys. 2004, 121, 6317.

(29) Even, U.; Jortner, J.; Noy, D.; Lavie, N.; Cossart-Magos, C. J. Chem. Phys. 2000, 112, 8068.

(30) Marcinow, Z.; Clawson, D. K.; Rabideau, P. W. Tetrahedron 1989, 45, 5441.

(31) DePuy, C. H.; Bierbaum, V. M.; Flippin, L. A.; Grabowski, J. J.; King, G. K.; Schmitt, R. J.; Sullivan, S. A. J. Am. Chem. Soc. 1980, 102, 5012.

(32) Kim, J. B.; Weichman, M. L.; Yacovitch, T. I.; Shih, C.; Neumark, D. M. J. Chem. Phys. 2013, 139, 104301.

(33) Gilbert, A. T. B.; Besley, N. A.; Gill, P. M. W. J. Phys. Chem. A 2008, 112, 13164.

(34) Shao, Y.; Molnar, L. F.; Jung, Y.; Kussmann, J.; Ochsenfeld, C.; Brown, S. T.; Gilbert, A. T. B.; Slipchenko, L. V.; Levchenko, S. V.; O'Neill, D. P.; DiStasio, R. A.; Lochan, R. C.; Wang, T.; Beran, G. J. O.; Besley, N. A.; Herbert, J. M.; Lin, C. Y.; Van Voorhis, T.; Chien, S. H.; Sodt, A.; Steele, R. P.; Rassolov, V. A.; Maslen, P. E.; Korambath, P. P.; Adamson, R. D.; Austin, B.; Baker, J.; Byrd, E. F. C.; Dachsel, H.; Doerksen, R. J.; Dreuw, A.; Dunietz, B. D.; Dutoi, A. D.; Furlani, T. R.; Gwaltney, S. R.; Heyden, A.; Hirata, S.; Hsu, C.-P.; Kedziora, G.; Khalliulin, R. Z.; Klunzinger, P.; Lee, A. M.; Lee, M. S.; Liang, W.; Lotan, I.; Nair, N.; Peters, B.; Proynov, E. I.; Pieniazek, P. A.; Rhee, Y. M.; Ritchie, J.; Rosta, E.; Sherrill, C. D.; Simmonett, A. C.; Subotnik, J.
E.; Woodcock, H. L.; Zhang, W.; Bell, A. T.; Chakraborty, A. K.; Chipman, D. M.; Keil, F. J.; Warshel, A.; Hehre, W. J.; Schaefer, H. F.; Kong, J.; Krylov, A. I.; Gill, P. M. W.; Head-Gordon, M. Phys. Chem. Chem. Phys. 2006, 8, 3172.

(35) Krylov, A. I.; Gill, P. M. W. WIREs Comput. Mol. Sci. 2013, 3, 317.

(36) Mozhayskiy, V. A.; Krylov, A. I. ezSpectrum 3.0, http:// iopenshell.usc.edu/downloads.

(37) Tao, L.; Oana, C. M.; Mozhayskiy, V. A.; Krylov, A. I. ezDyson, http://iopenshell.usc.edu/downloads/ezdyson.

(38) Oana, C. M.; Krylov, A. I. J. Chem. Phys. 2009, 131, 124114.

(39) Wigner, E. P. Phys. Rev. 1948, 73, 1002.

(40) Mabbs, R.; Grumbling, E. R.; Pichugin, K.; Sanov, A. Chem. Soc. Rev. 2009, 38, 2169.

(41) Culberson, L. M.; Blackstone, C. C.; Sanov, A. J. Phys. Chem. A 2013, 117, 11760. 